\title{
A Empatia em Acadêmicos de Medicina em Relação ao Paciente Pediátrico: Estudo Transversal Unicêntrico, 2019
}

\author{
Empathy in Medical Students in Relation to Pedriatic Patients: a \\ Cross-Sectional Study, 2019
}

Naiara Silva Cosmo de Araújo ${ }^{1}$ iD

Antonio Toledo Júnior ${ }^{\mathrm{I}}$

\section{PALAVRAS-CHAVE}

- Empatia.

- Educação Médica.

- Pediatria

Introdução: A empatia envolve a capacidade de identificar e perceber os sentimentos e as experiências pessoais do paciente, assim como a capacidade de ver o mundo da perspectiva de outra pessoa. Em pediatria, existe o desafio de buscar o bom relacionamento com o paciente pediátrico e seus acompanhantes, os quais são peças fundamentais no atendimento e no resultado do tratamento. O objetivo desse estudo foi avaliar o nível de empatia dos estudantes de Medicina em relação ao paciente pediátrico. Método: Trata-se de estudo transversal, com amostra de conveniência, realizado com estudantes do quarto e sexto anos do curso de Medicina da Universidade José do Rosário Vellano, Campus Alfenas. Os níveis de empatia foram avaliados por meio da Escala de Empatia de Jefferson. Questionário sociodemográfico coletou dados sobre as possíveis variáveis independentes, como sexo, idade, estado civil, doença crônica pessoal ou familiar, distúrbio mental, especialidade pretendida, entre outros. Utilizaram-se o teste de $t$ de Student, o teste exato de Fischer bicaudal e a regressão linear. O nível de significância foi de 0,05. Resultados: No período de fevereiro a abril de 2019, dos 196 estudantes elegíveis para o estudo, 159 concordaram em participar. Desses, 10 (6,2\%) foram excluídos por preenchimento incompleto do questionário. A amostra final foi de 149 (76,0\%) estudantes, sendo 74 (49,7\%) do quarto ano e 75 (50,3\%) do sexto ano do curso de Medicina. Não houve diferença entre o escore global de empatia entre o quarto e o sexto ano. O escore global de empatia foi maior no sexo feminino $\left(p_{\text {ajustado }}=0,011\right)$, assim como o escore do domínio "cuidado compassivo" ( $p_{\text {ajustado }}=0,013$ ). Os voluntários com relato de doença crônica familiar e os que tinham dificuldade de realizar exame físico pediátrico apresentaram escore significativamente mais elevado no domínio "cuidado compassivo" ( $p_{\text {ajustado }}=0,038$ e $p_{\text {ajustado }}=0,037$, respectivamente). Conclusões: Os níveis de empatia (escore global e diferentes domínios) observados neste estudo foram elevados, com exceção do domínio "capacidade de se colocar no lugar do outro". Não se observou relação entre escore global de empatia e seus diferentes domínios e o ano do curso médico. 


\section{KEYWORDS}

- Empathy.

- Medical Education.

- Pediatrics.

\section{ABSTRACT}

Introduction: Empathy involves the ability to identify and perceive a patient's personal feelings and experiences, as well as the ability to see the world from another person's perspective. In Pediatrics, there is the challenge of looking for a good relationship with the patient and their caregivers, who play a key role in both the care and the result of the treatment. The aim of this study was to evaluate the level of empathy of medical students related to pediatric patients. Methods: Cross-sectional study, with a convenience sample, conducted with $4^{\text {th }}$ and $6^{\text {th }}$-year medicine students at Universidade José do Rosário Vellano, Campus Alfenas. Levels of empathy were assessed using the Jefferson Scale of Empathy. A sociodemographic questionnaire collected data on possible independent variables such as gender, age, marital status, chronic individual and familial diseases, mental disorders and intended medical specialty. Student's $T$ test, two-tailed Fischer exact test and linear regression were used in statistical analysis. The level of significance was 0.05. Results: From February to April 2019, 159 students, from a total of 196 eligible candidates, agreed to participate in the study. Of these, 10 were excluded due to incomplete questionnaires. The final sample was composed of 149 (76.0\%) students, 74 (49.7\%) from the $4^{\text {th }}$-year and 75 (50.3\%) from the $6^{\text {th }}$-year. There was no difference between the overall empathy score between the $4^{\text {th }}$ and $6^{\text {th }}$-year students. The overall empathy score was higher in females $\left(p_{\text {adjusted }}=0.011\right)$, as was the Compassionate Care domain score $\left(p_{\text {adjusted }}=0.013\right)$. Volunteers reporting chronic familial disease and those who had difficulty performing a pediatric physical examination reported a significantly higher score in the Compassionate Care domain ( $p_{\text {adjusted }}=0.038$ and $p_{\text {adjusted }}=0.037$, respectively). Conclusion: The global empathy score and its different domains were high, except for the Ability to Stand in the Patient's Shoes domain. No relationship was found between empathy and its different domains and the year of undergraduate medical training.

Recebido em 13/5/20

Aceito em 13/5/20

\section{INTRODUÇÃO}

Nos longos anais da história médica, a pediatria é uma especialidade jovem, com pouco mais de 200 anos $^{1}$. No final dos anos 1700 e início dos anos 1800, a necessidade de atender especificamente ao cuidado, ao desenvolvimento e às doenças das crianças tornou-se mais aparente, e a especialização em pediatria evoluiu, particularmente na Alemanha e na França ${ }^{1}$. No Brasil, a especialidade teve grande impulso a partir 1910, quando foi criada a Sociedade Brasileira de Pediatria, associação científica voltada para o estudo de problemas e doenças infantis ${ }^{2}$.

$\mathrm{Na}$ pediatria, existe o desafio de buscar o bom relacionamento com o paciente e seus acompanhantes ou seus cuidadores, que são peças fundamentais na obtenção de informações durante a anamnese. Como os familiares são as principais fontes de força e apoio das crianças, devem ser valorizados ${ }^{3}$. Pensa-se que o bem-estar de um depende do bem-estar do outro, e, assim, há preocupação com a qualidade de vida da família e não apenas da criança ${ }^{4}$.

A relação médico-paciente é uma complexa interação psicossocial de vulnerabilidade, confiança e autoridade em ambiente profissional. Apesar de todo avanço tecnológico, ela continua a ser a pedra angular do cuidado: o meio no qual os dados são coletados, os diagnósticos e planos são feitos, a conformidade é realizada e a cura, a ativação do paciente e o suporte são fornecidos ${ }^{5,6}$.

No cenário de atendimento pediátrico, a relação médico-paciente tem se tornado assunto de grande importância, pois é necessário compreender a situação, a perspectiva e os sentimentos dos pacientes muitas vezes relatados pelos seus familiares ou seus cuidadores. A empatia torna-se então habilidade essencial no estabelecimento e na manutenção dessa relação ${ }^{3}$.

O termo empatia, do grego empatheia, significa a apreciação do sentimento do outro. Em seu sentido mais amplo, empatia pode ser definida como as reações cognitivas e emocionais de um indivíduo frente as experiências do outro ${ }^{7}$. Em Medicina, empatia envolve a capacidade de identificar e perceber os pensamentos e sentimentos do doente e tomar a perspectiva do doente acerca do problema apresentado ${ }^{8}$. Ela remete à sensibilização do médico pelas mudanças sentidas e refletidas momento a momento pelo paciente ${ }^{9}$. A sua vertente cognitiva engloba a capacidade de perceber os sentimentos e as experiências pessoais de cada paciente ("tomada de perspectiva"), assim como a capacidade de ver o mundo da perspectiva de outra pessoa ("capacidade de se colocar no lugar do outro") e a compaixão ("cuidado compassivo")

A empatia e a comunicação em consultas pediátricas possuem características peculiaridades comparadas aos adultos, visto que a criança está em processo de desenvolvimento cognitivo, social e psicológico ${ }^{15}$. Há preocupação crescente com a falta de empatia na prática clínica, agravada pela indicação da literatura de que ela diminui ao longo do curso de Medicina $^{13}$. Em revisão de literatura, Neumann et al. ${ }^{16}$ analisaram 18 artigos sobre o tema, dos quais 11 investigaram a empatia durante o curso de Medicina e sete estudaram a empatia durante a residência médica. Nenhum dos 18 estudos revelou aumento na empatia autoavaliada entre formandos em Medicina. Dois dos estudos mostraram aumento da empatia durante os primeiros anos de curso, mas houve declínio significativo na fase de prática clínica do curso, quando os alunos tiveram contato com pacientes ${ }^{16}$.

Os estudantes de Medicina experimentam mudanças de comportamento ao longo do curso, em decorrência da convivência com a doença, o sofrimento e a dificuldade em prover assistência adequada aos pacientes. Isso reduz o idealismo inicial e resulta em afastamento do paciente como mecanismo de defesa, contribuindo para a desumanização da assistência ${ }^{17}$. É interessante notar que a magnitude do declínio parece

REVISTA BRASILEIRA DE EDUCAÇÃO MÉDICA

2 44 (3) : e089; 2020 
ser maior entre os homens em comparação com as mulheres e entre aqueles que seguem carreiras voltadas para a tecnologia em comparação com os que optam por especialidades orientadas para as pessoas ${ }^{13}$.

O declínio da empatia pode ser associado a aspectos relativos à pressão e ao estresse mais frequentes com o avanço do curso, momento em que os alunos são sobrecarregados com atividades e responsabilidades, o que pode contribuir para a perda da qualidade de vida e ocasionar o esgotamento emocional ou burnout. Outros fatores que dificultam o desenvolvimento da empatia se referem à falta de atenção a esse aspecto nos cursos de Medicina, a interações negativas com pares e/ou professores $\mathrm{e}$ às condições adversas de trabalho ${ }^{18}$.

Em resumo, as relações empáticas em pediatria são essenciais para alcançar uma boa relação médico-paciente e bons resultados. $\mathrm{O}$ atendimento pediátrico envolve a tríade médico-paciente-acompanhante e necessita de relação de confiança e respeito, que facilita o fluxo de informações e explicações, resultando em melhora clínica e melhor adesão ao tratamento. Sendo assim, deve-se estudar e compreender o papel da empatia nesse contexto e compreender melhor os fatores relacionados ao seu aumento ou declínio, o que permitirá planejar estratégias de ensino capazes de melhorar a empatia de estudantes e médicos em relação ao paciente pediátrico e seus acompanhantes. Não foram identificados estudos que analisassem a empatia de estudantes de Medicina e médicos em relação ao paciente pediátrico na revisão da literatura. Portanto, é importante conhecer melhor essa realidade. Os objetivos desse trabalho foram avaliar o nível de empatia em estudantes de Medicina em relação ao paciente pediátrico e identificar fatores associados à empatia nesse contexto.

\section{MÉTODOS}

Trata-se de um estudo transversal desenvolvido no curso de Medicina da Universidade José do Rosário Vellano, Campus Alfenas.

\section{População}

A população-alvo foi constituída por 96 alunos do quarto ano e 100 do sexto ano do curso de Medicina. No referido curso, as disciplinas de Semiologia Pediátrica e Ambulatório de Pediatria ocorrem no terceiro ano e o Internato de Pediatria no quinto. Utilizou-se amostra não probabilística de conveniência. Os alunos foram recrutados por convites presenciais em sala de aula, reuniões da Liga de Pediatria ou e-mail.

O critério de inclusão foram: ser aluno de Medicina do quarto ou sexto ano do curso, estar regularmente matriculado e ter concluído as respectivas disciplinas de pediatria. Foram excluídos todos os alunos reprovados em alguma das disciplinas de pediatria e aqueles com a matrícula trancada ou afastados por outros motivos. Todos os voluntários assinaram o Termo de Consentimento Livre e Esclarecido (TCLE) antes de sua inclusão no estudo.

\section{Procedimentos}

Como nenhum instrumento específico de empatia em pediatria foi identificado na literatura, optou-se por utilizar a Escala de Empatia de Jefferson (EEJ), versão adaptada para a população brasileira por Paro et al. ${ }^{19}$, e um questionário sociodemográfico elaborado pelos autores.

A EEJ permite avaliar a percepção do estudante acerca do seu comportamento empático na prestação de cuidados ao paciente e da relevância da qualidade da relação médico-paciente ${ }^{13}$. Ela avalia $o$ aspecto cognitivo da empatia. Contém 20 itens em escala do tipo Likert de 7 pontos (de 1 - discordo totalmente - a 7 - concordo fortemente). Para efeito de pontuação, 10 itens $(1,3,6,7,8,11,12,14,18,19)$ formulados na negativa são recodificados inversamente. Posteriormente, o valor da pontuação de cada item é somado. O escore global varia de 20 a 140 pontos. Quanto maior o escore total, maior é o nível de empatia ${ }^{10,19}$. A versão brasileira da EEJ possui três domínios: "tomada de perspectiva" (sete itens: 4, 5, 9, 10, 13, 17 e 18), "cuidado compassivo" (11 itens: 1, 2, $7,8,11,12,14,15,16,19$ e 20$)$ e "capacidade de se colocar no lugar do outro" (dois itens: 3 e 6 ) ${ }^{19}$.

O questionário sociodemográfico foi desenvolvido com o objetivo de avaliar variáveis que possam influenciar a empatia, como idade, sexo, filhos, irmãos, número de irmãos, possuir irmãos mais novos, estado civil, opção de especialidade, ter graduação prévia, doença grave pessoal ou familiar, diagnóstico prévio de doença mental (autorrelato) e a relação com pacientes pediátricos. Utilizaram-se também perguntas que enfatizavam o relacionamento com paciente pediátrico:

- Gosta de atender crianças?

- Acha difícil o exame físico na criança?

- Gosta de estudar doenças pediátricas?

- Acha importante ter um contato próximo com o paciente pediátrico em ambulatórios, pronto atendimento e setores de emergência?

- Já teve contato com paciente pediátrico grave?

- Sente-se à vontade para conversar com os responsáveis dos pacientes pediátricos?

Antes da aplicação dos questionários, ressaltou-se que eles deveriam ser preenchidos pensando no atendimento pediátrico e certificou-se de que essa orientação foi bem entendida pelos voluntários.

\section{Análise estatística}

O desfecho principal foi o escore global de empatia e os desfechos secundários os níveis dos diferentes domínios de empatia medidos pela EEJ. A principal variável de exposição foi o ano do curso de Medicina, dividido em dois grupos: quarto ano e sexto ano. $\mathrm{O}$ banco de dados foi construído utilizando-se o software Remark Office OMR (Remark, USA) e a análise estatística feita pelo software $\mathrm{IBM}^{\circ}$ SPSS $^{\circledR}$ Statistics v. 19 (IBM, USA). Antes da análise estatística, foi feita conferência manual do banco de dados e conferência por amplitude e lógica, para garantir a integridade dos dados. O escore global e os escores dos domínios foram calculados por meio do SPSS ${ }^{\circledR}$.

A análise descritiva utilizou média e desvio padrão para variáveis contínuas e distribuição de frequência para variáveis categóricas. Utilizouse o teste $\mathrm{t}$ de Student para comparação dos escores de empatia e as diversas variáveis contínuas. O teste exato de Fisher bicaudal foi utilizado na comparação com variáveis categóricas. Realizou-se análise de regressão linear entre as variáveis que apresentaram $\mathrm{p}<0,25$ na análise bivariada. Optou-se pelo valor de corte de 0,25 por ser uma abordagem mais conservadora, que permitiu a inclusão de número maior de variáveis e avaliação de mais interações potenciais do que o valor tradicional de 0,20. Considerou-se o nível de significância de 0,05.

\section{Aspectos éticos}

Este trabalho foi aprovado pelo Comitê de Ética em Pesquisa em Seres

REVISTA BRASILEIRA DE EDUCAÇÃO MÉDICA

3 $44(3)$ : e089; 2020 
Humanos da Universidade José do Rosário Vellano (Parecer n 3.065.732). Todos participantes assinaram o TCLE antes de sua inclusão e todos os esforços foram feitos para a manutenção do sigilo das informações.

\section{RESULTADOS}

No período de fevereiro a abril de 2019, 159 estudantes concordaram em participar do estudo e assinaram o TCLE. Desses, 10 (6,2\%) foram excluídos por preenchimento incompleto do questionário. A amostra final foi de 149 estudantes, sendo 74 (49,7\%) do quarto ano e 75 (50,3\%) do sexto ano do curso de Medicina. Esse total representou 76,0\% dos 196 alunos que preenchiam o critério de inclusão, sendo $77,1 \%$ dos alunos elegíveis do quarto ano (74/96) e 75,0\% dos alunos elegíveis do sexto ano (75/100).

A Tabela 1 apresenta as características sociodemográficas, pessoais e relacionadas ao atendimento pediátrico dos 149 estudantes de acordo com o ano do curso. Observa-se predomínio de pessoas do sexo feminino (91 -

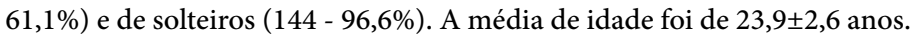
Apenas dois (1,9\%) participantes tinham filhos. Dez (6,7\%) apresentavam graduação prévia. A maior parte deles (134 - 89,9\%) relatou ter irmãos e $75(56,0 \%)$ tinham irmãos mais novos. Setenta e oito $(58,2 \%)$ participantes tinham apenas um irmão; $39(29,1 \%)$, dois; e $17(12,7 \%)$, três ou mais. Vinte e quatro $(16,1 \%)$ estudantes relataram doença crônica pessoal. Percentual significativamente maior de estudantes do sexto ano relatou doença crônica familiar $(46,7 \%$ versus $25,6 \% ; \mathrm{p}=0,010)$. Os distúrbios mentais autorrelatados também foram significativamente mais frequentes entre estudantes do sexto ano (18,7\% versus $6,8 \% ; \mathrm{p}=0,047)$. Apesar de apenas $16(10,7 \%)$ participantes pretenderem fazer pediatria, mais da metade (101 - 67,8\%) afirmou gostar de atender crianças e $104(70,3 \%)$ de estudar doenças pediátricas. A maior parte dos participantes (101-67,8\%) achava difícil realizar o exame físico na criança, 143 (96,6\%) consideravam importante ter contato com paciente pediátrico durante a graduação e $125(83,9 \%)$ ficavam à vontade para conversar com os responsáveis dos pacientes pediátricos durante atendimento. $\mathrm{O}$ percentual de alunos que teve contato com paciente pediátrico grave foi significativamente maior no sexto ano $(76,0 \%$ versus $25,7 \%, \mathrm{p}<0,001)$.

A Tabela 2 ilustra o escore global e por domínios de empatia de acordo com o ano do curso. Não se observou diferenças significativas entre os dois anos. Os escores global e dos domínios "cuidado compassivo" e "tomada de perspectiva" foram elevados, representando 84,6\%, 91,6\% e $81,4 \%$ do total de pontos possíveis, respectivamente. Já o escore do domínio "capacidade de se colocar no lugar do outro" representou 63,6\% do total possível.

A Tabela 3 demonstra a média e o desvio padrão do escore global da escala de Jefferson de acordo com outras variáveis. De modo geral, não houve diferenças significativas entre as diferentes variáveis analisadas, exceto em relação ao sexo. As mulheres apresentaram escore global significativamente mais elevado do que os homens $(120,4 \pm 9,2$ versus $115,4 \pm 12,2 ; \mathrm{p}=0,005)$.

A Tabela 4 mostra a comparação entre os domínios "cuidado compassivo" e "capacidade de se colocar no lugar do outro" e as demais variáveis. O escore de "cuidado compassivo" foi significativamente mais elevado no sexo feminino $(71,9 \pm 5,6$ versus $68,3 \pm 7,9 ; \mathrm{p}=0,030)$ e naqueles que relataram possuir doença crônica na família $(72 \pm 5,0$ versus $69,7 \pm 7,6$; $\mathrm{p}=0,027)$. É interessante destacar que os estudantes que relataram achar o exame físico na criança difícil apresentaram escores mais elevados, 71,4 66,0

\begin{tabular}{|c|c|c|c|c|c|c|c|}
\hline \multicolumn{8}{|c|}{$\begin{array}{c}\text { Tabela } 1 \\
\text { Características sociodemográficas, pessoais e relacionadas ao } \\
\text { atendimento pediátrico dos } 149 \text { estudantes de acordo com ano do curso }\end{array}$} \\
\hline \multirow{2}{*}{\multicolumn{2}{|c|}{ Variável }} & \multicolumn{2}{|c|}{$\begin{array}{c}4^{\circ} \text { ano } \\
(n=74)\end{array}$} & \multicolumn{2}{|c|}{$\begin{array}{c}6^{\circ} \text { ano } \\
(n=75)\end{array}$} & \multicolumn{2}{|c|}{$\begin{array}{c}\text { Total } \\
(\mathbf{n}=149)\end{array}$} \\
\hline & & $\mathbf{N}$ & $\%$ & $\mathbf{N}$ & $\%$ & $\mathbf{n}$ & $\%$ \\
\hline \multirow{2}{*}{ Sexo } & Masculino & 29 & 39,2 & 29 & 38,7 & 58 & 38,9 \\
\hline & Feminino & 45 & 60,8 & 46 & 61,3 & 91 & 61,1 \\
\hline \multirow{3}{*}{ Estado civil } & Solteiro & 73 & 98,6 & 71 & 94,7 & 144 & 96,6 \\
\hline & Casado & 0 & 0,0 & 4 & 5,3 & 4 & 2,7 \\
\hline & Separado & 1 & 1,4 & 0 & 0,0 & 1 & 0,7 \\
\hline \multirow{2}{*}{ Filhos } & Sim & 2 & 2,7 & 0 & 0,0 & 2 & 1,3 \\
\hline & Não & 72 & 97,3 & 75 & 100 & 147 & 98,7 \\
\hline \multirow{2}{*}{ Graduação prévia } & Sim & 5 & 6,8 & 5 & 6,7 & 10 & 6,7 \\
\hline & Não & 69 & 93,2 & 70 & 93,3 & 139 & 93,3 \\
\hline \multirow{2}{*}{ Tem irmãos } & Sim & 68 & 91,9 & 66 & 88,0 & 134 & 89,9 \\
\hline & Não & 6 & 8,1 & 9 & 12,0 & 15 & 10,1 \\
\hline \multirow{2}{*}{$\begin{array}{l}\text { Tem irmãos mais } \\
\text { novos }\end{array}$} & Sim & 36 & 52,9 & 39 & 59,1 & 75 & 56,0 \\
\hline & Não & 32 & 47,1 & 27 & 40,9 & 59 & 44,0 \\
\hline \multirow{3}{*}{ Número de irmãos } & 1 & 38 & 55,9 & 40 & 60,6 & 78 & 58,2 \\
\hline & 2 & 23 & 33,8 & 16 & 24,2 & 39 & 29,1 \\
\hline & $>3$ & 7 & 10,3 & 10 & 15,2 & 17 & 12,7 \\
\hline \multirow{2}{*}{$\begin{array}{l}\text { Doença crônica } \\
\text { pessoal }\end{array}$} & Sim & 11 & 14,9 & 13 & 17,3 & 24 & 16,1 \\
\hline & Não & 63 & 85,1 & 62 & 82,7 & 125 & 83,9 \\
\hline \multirow{2}{*}{$\begin{array}{l}\text { Doença crônica } \\
\text { familiar }^{*}\end{array}$} & Sim & 19 & 25,7 & 35 & 46,7 & 54 & 36,2 \\
\hline & Não & 55 & 74,3 & 40 & 53,3 & 95 & 63,8 \\
\hline \multirow{2}{*}{$\begin{array}{l}\text { Distúrbio mental } \\
\text { prévio }^{* *}\end{array}$} & Sim & 5 & 6,8 & 14 & 18,7 & 19 & 12,8 \\
\hline & Não & 69 & 93,2 & 61 & 81,3 & 130 & 87,2 \\
\hline \multirow{2}{*}{$\begin{array}{l}\text { Pretende fazer } \\
\text { pediatria? }\end{array}$} & Sim & 7 & 9,5 & 9 & 12,0 & 16 & 10,7 \\
\hline & Não & 67 & 90,5 & 66 & 88,0 & 133 & 89,3 \\
\hline \multirow{2}{*}{$\begin{array}{l}\text { Gosta de atender } \\
\text { crianças? }\end{array}$} & Sim & 49 & 66,2 & 52 & 69,3 & 101 & 67,8 \\
\hline & Não & 25 & 33,8 & 23 & 30,7 & 48 & 32,2 \\
\hline \multirow{2}{*}{$\begin{array}{l}\text { Gosta de estudar } \\
\text { doenças } \\
\text { pediátricas? }\end{array}$} & Sim & 48 & 64,9 & 56 & 75,7 & 104 & 70,3 \\
\hline & Não & 26 & 35,1 & 18 & 24,3 & 44 & 29,7 \\
\hline \multirow{2}{*}{$\begin{array}{l}\text { Acha difícil fazer } \\
\text { exame físico na } \\
\text { criança? }\end{array}$} & Sim & 55 & 74,3 & 46 & 61,3 & 101 & 67,8 \\
\hline & Não & 19 & 25,7 & 29 & 38,7 & 48 & 32,2 \\
\hline \multirow{2}{*}{$\begin{array}{l}\text { Já teve contato com } \\
\text { paciente pediátrico } \\
\text { grave?***}\end{array}$} & Sim & 19 & 25,7 & 57 & 76,0 & 76 & 51,0 \\
\hline & Não & 55 & 74,3 & 18 & 24,0 & 73 & 49,0 \\
\hline \multirow{2}{*}{$\begin{array}{l}\text { Acha importante ter } \\
\text { contato com } \\
\text { paciente pediátrico? }\end{array}$} & Sim & 71 & 97,3 & 72 & 96,0 & 143 & 96,6 \\
\hline & Não & 2 & 2,7 & 3 & 4,0 & 5 & 3,4 \\
\hline \multirow{2}{*}{$\begin{array}{l}\text { Sente-se à vontade } \\
\text { para conversar com } \\
\text { os responsáveis dos } \\
\text { pacientes pediátricos? }\end{array}$} & Sim & 62 & 83,8 & 63 & 84,0 & 125 & 83,9 \\
\hline & Não & 12 & 16,2 & 12 & 16,0 & 24 & 16,1 \\
\hline
\end{tabular}

${ }^{*} \mathrm{p}=0,010 ;{ }^{* *} \mathrm{p}=0,047 ;{ }^{* * *} \mathrm{p}<0,001$.

Fonte: elaborada pelos autores 


\begin{tabular}{|c|c|c|c|c|c|c|c|}
\hline Escore global d & $\begin{array}{r}\text { empatia } \\
\text { acordo }\end{array}$ & $\begin{array}{l}\text { Tabe } \\
\text { por } \\
\text { com }\end{array}$ & $\begin{array}{l}\text { a } 2 \\
\text { lomínio } \\
\text { no do } \mathrm{cl}\end{array}$ & $\begin{array}{l}\text { de } 14 \\
\text { rso }\end{array}$ & 9 estuda & ates $\mathrm{c}$ & \\
\hline Variável & $\begin{array}{l}4^{\circ} \text { a } \\
(n=)\end{array}$ & & $\begin{array}{l}6^{0} a \\
(n=\end{array}$ & & $\begin{array}{r}\text { Tot: } \\
(n=1\end{array}$ & & $\mathbf{p}$ \\
\hline & Média & DP & Média & DP & Média & DP & \\
\hline Escore global & 118,1 & 15,1 & 118,8 & 15,5 & 118,5 & 15,3 & 0,701 \\
\hline Cuidado compassivo & 70,2 & 6,8 & 70,8 & 6,9 & 70,5 & 6,8 & 0,604 \\
\hline $\begin{array}{l}\text { Tomada de } \\
\text { perspectiva }\end{array}$ & 38,9 & 5,3 & 39,1 & 5,5 & 39,9 & 5,4 & 0,881 \\
\hline $\begin{array}{l}\text { Capacidade de se } \\
\text { colocar no lugar } \\
\text { do outro }\end{array}$ & 8,9 & 3,0 & 8,9 & 3,1 & 8,9 & 3,0 & 0,939 \\
\hline
\end{tabular}

DP: desvio padrão.

Fonte: elaborada pelos autores

\begin{tabular}{|c|c|c|c|c|c|}
\hline \multicolumn{6}{|c|}{$\begin{array}{c}\text { Tabela } 3 \\
\text { Média e desvio padrão do escore global da Escala de Jefferson de } \\
\text { acordo com as características pessoais de } 149 \text { estudantes de Medicina }\end{array}$} \\
\hline \multirow{2}{*}{ Variável } & \multicolumn{2}{|c|}{ Feminino } & \multicolumn{2}{|c|}{ Masculino } & \multirow[b]{2}{*}{$\mathbf{p}$} \\
\hline & Média & DP & Média & DP & \\
\hline Sexo & 120,4 & 9,2 & 115,4 & 12,2 & $\underline{0,005}$ \\
\hline \multirow{2}{*}{ Variável } & \multicolumn{2}{|c|}{ Sim } & \multicolumn{2}{|c|}{ Não } & \\
\hline & Média & DP & Média & $\mathrm{DP}$ & $\mathrm{p}$ \\
\hline Tem outra graduação? & 119,5 & 10,9 & 118,4 & 10,7 & 0,753 \\
\hline Tem irmãos? & 118,2 & 10,8 & 121,0 & 10,2 & 0,336 \\
\hline Tem irmão(s) mais novo(s)? & 117,3 & 11,1 & 119,3 & 10,4 & 0,292 \\
\hline $\begin{array}{l}\text { Possui algum tipo de doença } \\
\text { crônica pessoal? }\end{array}$ & 121,3 & 10,8 & 117,9 & 10,7 & 0,159 \\
\hline $\begin{array}{l}\text { Possui algum tipo de doença } \\
\text { crônica familiar? }\end{array}$ & 119,5 & 9,3 & 117,8 & 11,5 & 0,358 \\
\hline $\begin{array}{l}\text { Já teve diagnóstico prévio de } \\
\text { algum distúrbio psiquiátrico? }\end{array}$ & 119,4 & 10,2 & 118,3 & 10,8 & 0,678 \\
\hline Pretende fazer pediatria? & 120,6 & 7,5 & 118,2 & 11,0 & 0,409 \\
\hline Gosta de atender crianças? & 118,5 & 10,1 & 118,3 & 12,0 & 0,932 \\
\hline $\begin{array}{l}\text { Gosta de estudar doenças } \\
\text { pediátricas? }\end{array}$ & 118,3 & 10,4 & 118,6 & 11,6 & 0,880 \\
\hline $\begin{array}{l}\text { Acha difícil fazer exame físico na } \\
\text { criança? }\end{array}$ & 119,0 & 9,6 & 117,2 & 12,8 & 0,351 \\
\hline $\begin{array}{l}\text { Já teve contato com paciente } \\
\text { pediátrico grave? }\end{array}$ & 118,7 & 11,0 & 118,2 & 10,5 & 0,787 \\
\hline $\begin{array}{l}\text { Acha importante ter contato } \\
\text { com paciente pediátrico em } \\
\text { ambulatórios, pronto atendimento } \\
\text { e emergência? }\end{array}$ & 118,2 & 10,4 & 123,2 & 17,3 & 0,301 \\
\hline $\begin{array}{l}\text { Sente-se à vontade para conversar } \\
\text { com os responsáveis (exemplo: } \\
\text { pais, avós) dos pacientes } \\
\text { pediátricos? }\end{array}$ & 118,0 & 10,6 & 120,5 & 11,3 & 0,311 \\
\hline
\end{tabular}

DP: desvio padrão.

Fonte: elaborada pelos autores

\section{Tabela 4}

Média e desvio padrão de diferentes domínios da Escala de Jefferson de acordo com as variáveis de 149 estudantes de Medicina

\begin{tabular}{|c|c|c|c|c|c|}
\hline \multirow{2}{*}{ Cuidado compassivo } & \multicolumn{2}{|c|}{ Feminino } & \multicolumn{2}{|c|}{ Masculino } & \multirow[b]{2}{*}{ p } \\
\hline & Média & DP & Média & DP & \\
\hline Sexo & 71,9 & 5,6 & 68,3 & 7,9 & $\underline{0,030}$ \\
\hline \multirow{2}{*}{ Variável } & \multicolumn{2}{|c|}{ Sim } & \multicolumn{2}{|c|}{ Não } & \\
\hline & Média & $\mathrm{DP}$ & Média & $\mathrm{DP}$ & $\mathrm{p}$ \\
\hline Tem outra graduação? & 69,7 & 5,6 & 70,6 & 6,9 & 0,685 \\
\hline Tem irmãos? & 70,4 & 7,0 & 72,0 & 4,1 & 0,366 \\
\hline Tem irmão(s) mais novo(s)? & 70,0 & 7,8 & 71,1 & 5,6 & 0,312 \\
\hline Possui algum tipo de doença crônica pessoal? & 71,7 & 5,6 & 70,3 & 7,0 & 0,349 \\
\hline Possui algum tipo de doença crônica familiar? & 72,0 & 5,0 & 69,7 & 7,6 & $\underline{\mathbf{0 , 0 2 7}}$ \\
\hline $\begin{array}{l}\text { Já teve diagnóstico prévio de algum } \\
\text { distúrbio psiquiátrico? }\end{array}$ & 70,0 & 7,9 & 70,6 & 6,7 & 0,708 \\
\hline Pretende fazer pediatria? & 73,0 & 4,2 & 70,3 & 7,0 & 0,129 \\
\hline Gosta de atender crianças? & 70,7 & 6,5 & 70,1 & 7,4 & 0,584 \\
\hline Gosta de estudar doenças pediátricas? & 70,6 & 6,1 & 70,2 & 8,3 & 0,742 \\
\hline Acha difícil fazer exame físico na criança? & 71,4 & 6,0 & 68,8 & 8,0 & $\underline{0,050}$ \\
\hline Já teve contato com paciente pediátrico grave? & 71,0 & 6,5 & 70,0 & 7,2 & 0,414 \\
\hline $\begin{array}{l}\text { Acha importante ter contato com paciente } \\
\text { pediátrico em ambulatórios, pronto }\end{array}$ & 70,5 & 6,8 & 71,2 & 7,7 & 0,810 \\
\hline
\end{tabular}
atendimento e emergência?

\begin{tabular}{|c|c|c|c|c|c|}
\hline $\begin{array}{l}\text { Sente-se à vontade para conversar com } \\
\text { os responsáveis (exemplo: pais, avós) dos } \\
\text { pacientes pediátricos? }\end{array}$ & 70,3 & 6,8 & 71,7 & 6,9 & 0,366 \\
\hline \multirow{2}{*}{$\begin{array}{l}\text { Capacidade de se colocar no lugar do } \\
\text { outro }\end{array}$} & \multicolumn{2}{|c|}{ Feminino } & \multicolumn{2}{|c|}{ Masculino } & \\
\hline & Média & DP & Média & DP & $\mathbf{p}$ \\
\hline Sexo & 8,9 & 3,0 & 8,9 & 3,1 & 0,886 \\
\hline \multirow[b]{2}{*}{ Variável } & \multicolumn{2}{|c|}{ Sim } & \multicolumn{2}{|c|}{ Não } & \\
\hline & Média & DP & Média & $\mathrm{DP}$ & $\mathrm{p}$ \\
\hline Tem outra graduação? & 10,2 & 2,9 & 8,8 & 3,1 & 0,161 \\
\hline Tem irmãos? & 8,9 & 3,0 & 8,9 & 3,9 & 0,980 \\
\hline Tem irmão(s) mais novo(s)? & 8,3 & 2,8 & 9,4 & 3,2 & $\underline{0,026}$ \\
\hline Possui algum tipo de doença crônica pessoal? & 9,2 & 2,7 & 8,8 & 3,1 & 0,575 \\
\hline Possui algum tipo de doença crônica familiar? & 8,9 & 3,1 & 8,9 & 3,1 & 0,963 \\
\hline $\begin{array}{l}\text { Já teve diagnóstico prévio de algum } \\
\text { distúrbio psiquiátrico? }\end{array}$ & 9,0 & 3,3 & 8,9 & 3,0 & 0,863 \\
\hline Pretende fazer pediatria? & 8,4 & 2,5 & 8,9 & 3,1 & 0,537 \\
\hline Gosta de atender crianças? & 8,5 & 3,1 & 9,6 & 2,8 & $\underline{0,036}$ \\
\hline Gosta de estudar doenças pediátricas? & 9,0 & 3,0 & 8,8 & 3,1 & 0,764 \\
\hline Acha difícil fazer exame físico na criança? & 8,8 & 3,2 & 9,0 & 2,8 & 0,843 \\
\hline Já teve contato com paciente pediátrico grave? & 8,6 & 3,1 & 9,2 & 3,0 & 0,213 \\
\hline $\begin{array}{l}\text { Acha importante ter contato com paciente } \\
\text { pediátrico em ambulatórios, pronto } \\
\text { atendimento e emergência? }\end{array}$ & 8,8 & 3,1 & 11,4 & 2,9 & 0,059 \\
\hline
\end{tabular}

atendimento e emergência?

Sente-se à vontade para conversar com

os responsáveis (exemplo: pais, avós) dos $\quad \begin{array}{llllll}9,0 & 3,0 & 8,4 & 3,3 & 0,414\end{array}$ pacientes pediátricos?

DP: desvio padrão.

Fonte: elaborada pelos autores 
versus $68,8 \pm 8,0$; com $p=0,050$. Em relação ao domínio "capacidade de se colocar no lugar do outro", os estudantes que relataram ter irmãos mais novos apresentaram escore menor $(8,3 \pm 2,8$ versus $9,5 \pm 3,2 \mathrm{p}=0,026)$. Estudantes que não gostam de atender crianças apresentaram escores significativamente maiores $(9,6 \pm 2,8$ versus $8,5 \pm 3,1 ; \mathrm{p}=0,036)$ que os gostam. Não se observou nenhuma diferença significativa na comparação do domínio "tomada de perspectiva" com as variáveis sociodemográficas e pessoais e entre a idade e o escore global de empatia e de seus diferentes domínios (dados não apresentados).

$\mathrm{Na}$ análise multivariada, foram incluídas as variáveis $\operatorname{com} \mathrm{p}<0,25$ na análise bivariada. Também foram incluídas as variáveis sexo e "pretende fazer pediatria”, uma vez apenas mulheres indicaram a intenção de fazer a especialidade (dados não apresentados). A Tabela 5 apresenta os valores de $\mathrm{p}_{\text {bruto }}$ (análise bivariada) e $\mathrm{p}_{\text {ajustado }}$ pela análise multivariada. As mulheres apresentaram escore global estatisticamente superior $\left(\mathrm{p}_{\text {ajustado }}=0,009\right)$ aos homens, assim como no domínio "cuidado compassivo" ( $\mathrm{p}_{\text {ajustado }}=$ 0,013). Ainda nesse domínio, a relação entre escores mais elevados e a presença de doença crônica familiar e dificuldade de fazer o exame físico na criança permaneceu estatisticamente significativa, com $\mathrm{p}_{\text {ajustado }}=0,038$ e $\mathrm{p}_{\text {ajustado }}=0,037$, respectivamente. Nos outros dois domínios, "tomada de perspectiva" e "capacidade de se colocar no lugar do outro", nenhuma das variáveis mostrou significância estatística na análise multivariada.

\section{DISCUSSÃO}

Os resultados indicaram níveis elevados de empatia considerando-se o escore global, o "cuidado compassivo" e a "tomada de perspectiva". O domínio "capacidade de se colocar no lugar do outro" apresentou pontuação baixa. Esses resultados foram semelhantes aos observados por Nascimento et al. ${ }^{14}$, que avaliaram 152 alunos do último do curso de Medicina em Goiânia. Paro et al. ${ }^{19}$ também observaram níveis elevados em empatia em 296 estudantes de Medicina do último ano do curso em São Paulo.

Nesse estudo, não se observou diferença entre o escore global de empatia e seus domínios entre os estudantes do quarto ano e sexto ano. Esses achados diferem do trabalho de Hojat et al. ${ }^{13}$, que observaram declínio significativo nos escores de empatia ao final do terceiro ano, que persistiu até $\mathrm{o}$ fim da graduação. Já o estudo de Kataoka et al. ${ }^{20}$, que avaliou 400 estudantes da Faculdade de Medicina de Okayama em 2006 a 2007, evidenciou aumento da empatia ao longo do curso. Os autores indicaram que essa diferença pode estar relacionada a fatores culturais, à forma de ingresso na faculdade e ao currículo das escolas médicas no Japão. Por exemplo, o vestibular para Medicina no Japão é fortemente orientado para a ciência e não para disciplinas humanísticas e habilidades relacionadas ao profissionalismo, favorecendo níveis menores de empatia entre os alunos no início do curso. Durante o curso, são oferecidas disciplinas especiais, como história da medicina, bioética, vida e morte e habilidades de comunicação, motivando os alunos e proporcionando aumento da empatia $^{20}$. Segundo Magalhães et al. $^{21}$, o comportamento da empatia ao longo do curso médico pode variar muito de acordo com fatores culturais. Estudos transversais realizados no Japão e na Coreia mostram aumento dos níveis de empatia, enquanto os estudos norte-americanos mostram declínio. Dessa forma, a generalização do comportamento da empatia em escolas de Medicina é incerta ${ }^{21}$.

A análise multivariada indicou que os voluntários do sexo feminino apresentaram escores global $\left(\mathrm{p}_{\text {ajustado }}=0,011\right)$ e do domínio "cuidado

\begin{tabular}{|c|c|c|c|c|}
\hline $\begin{array}{r}\text { Regressão linear de } \\
\text { empa }\end{array}$ & ariáveis & $\begin{array}{l}\text { a } 5 \\
\text { cionadas por } \\
\text { rentes domín }\end{array}$ & score gl & al de \\
\hline Variável & $\begin{array}{r}\text { Coe } \\
\text { pa }\end{array}$ & $\begin{array}{l}\text { entes não } \\
\text { nizados }\end{array}$ & $\mathbf{p}_{\text {bruto }}$ & $\mathbf{p}_{\text {ajustado }}$ \\
\hline Escore total & Beta & Erro padrão & & \\
\hline Sexo & $-4,904$ & 1,842 & 0,005 & 0,011 \\
\hline Pretende fazer pediatria & 0,410 & 2,909 & 0,409 & 0,943 \\
\hline Doença crônica pessoal & 1,561 & 1,801 & 0,159 & 0,276 \\
\hline Cuidado compassivo & Beta & Erro padrão & $\mathbf{p}_{\text {bruto }}$ & $\mathbf{p}_{\text {ajustado }}$ \\
\hline Sexo & $-2,900$ & 1,153 & 0,030 & 0,013 \\
\hline Pretende fazer pediatria & 2,436 & 1,836 & 0,129 & 0,187 \\
\hline Doença crônica familiar & 2,316 & 1,108 & 0,027 & 0,038 \\
\hline $\begin{array}{l}\text { Acha difícil fazer exame } \\
\text { físico na criança }\end{array}$ & 2,470 & 1,173 & 0,050 & 0,037 \\
\hline Tomada de perspectiva & Beta & Erro padrão & $\mathbf{p}_{\text {bruto }}$ & $\mathbf{p}_{\text {ajustado }}$ \\
\hline Sexo & $-1,372$ & 0,954 & 0,124 & 0,153 \\
\hline Pretende fazer pediatria & $-0,497$ & 1,518 & 0,939 & 0,744 \\
\hline Doença crônica pessoal & 0,961 & 1,232 & 0,197 & 0,436 \\
\hline Distúrbio mental prévio & 1,683 & 1,346 & 0,230 & 0,213 \\
\hline $\begin{array}{l}\text { Sente-se à vontade } \\
\text { para conversar com } \\
\text { os responsáveis dos } \\
\text { pacientes pediátricos? }\end{array}$ & $-1,391$ & 1,235 & 0,183 & 0,153 \\
\hline $\begin{array}{l}\text { Capacidade de se colocar } \\
\text { no lugar do outro }\end{array}$ & Beta & Erro padrão & $\mathbf{p}_{\text {bruto }}$ & $\mathbf{p}_{\text {ajustado }}$ \\
\hline Sexo & $-1,457$ & 0,965 & 0,886 & 0,133 \\
\hline Pretende fazer pediatria & $-0,568$ & 1,547 & 0,537 & 0,714 \\
\hline Graduação prévia & 0,415 & 1,810 & 0,161 & 0,819 \\
\hline Tem irmão mais novo & 0,055 & 0,903 & 0,026 & 0,952 \\
\hline Gosta de atender crianças & 0,643 & 1,033 & 0,036 & 0,535 \\
\hline $\begin{array}{l}\text { Acha importante ter } \\
\text { contato com paciente } \\
\text { pediátrico em } \\
\text { ambulatórios, pronto } \\
\text { atendimento e } \\
\text { emergência }\end{array}$ & $-2,739$ & 2,591 & 0,059 & 0,292 \\
\hline
\end{tabular}

Fonte: elaborada pelos autores

compassivo" $\left(\mathrm{p}_{\text {ajustado }}=0,013\right)$ mais elevados. Esses achados são semelhantes a outros relados da literatura, que também indicam que as mulheres possuem níveis mais elevados de empatia que os homens ${ }^{8,10,12,13,16}$. Segundo o estudo de Nascimento et al. ${ }^{14}$, as mulheres tendem a estabelecer relações médico-paciente mais harmoniosas, colaborativas e democráticas. A mulher médica é capaz de entender com mais facilidade as particularidades e preferências de cada paciente, com maior cuidado compassivo ${ }^{14}$. Alguns autores sugerem que as mulheres são mais receptivas que os homens a sinais emocionais, qualidade que pode contribuir para melhor compreensão e, portanto, para relacionamento mais empático. Além disso, acredita-se que as mulheres desenvolvam atitudes mais cuidadoras em relação aos filhos

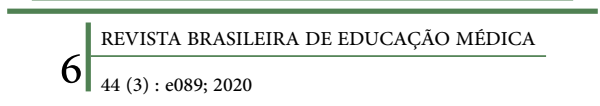


do que os homens. As descobertas sobre diferenças de sexo na empatia estão de acordo com relatos de que as médicas passam mais tempo com seus pacientes, têm menos pacientes e desempenham atendimentos mais preventivos e orientados aos pacientes ${ }^{14,16,22}$.

Outro ponto importante nesse estudo foi a relação dos níveis de empatia com a presença de doença crônica familiar, com escore significativamente mais alto para o domínio "cuidado compassivo" entre os voluntários que relataram essa situação. Estudo realizado com 191 estudantes em uma faculdade de Medicina na Espanha não encontrou associação entre empatia e doença pessoal ou familiar, mas identificou que alunos que tinham amigos doentes apresentavam escore global de empatia mais elevado ${ }^{23}$. Esse achado sugere que o contato com o doente crônico pode aumentar a capacidade de perceber problemas de outras pessoas.

A maior parte dos participantes desse estudo achou difícil realizar o exame físico na criança. É interessante observar que justamente os que relataram essa dificuldade apresentaram níveis mais elevados no domínio "cuidado compassivo", talvez por serem capazes de compreender as preocupações e os sentimentos em relação ao paciente pediátrico e aos seus acompanhantes, mesmo havendo dificuldades técnicas em realizar o exame físico.

As principais limitações deste estudo incluem o desenho transversal, a amostra de conveniência e o fato de ter sido realizado em apenas um curso de Medicina, o que pode comprometer a generalização dos resultados. A utilização de estudo transversal não permite avaliar as alterações que podem ocorrer ao longo do curso em determinado grupo de alunos. Sua utilização parte do princípio que a turma mais avançada representaria a evolução da turma menos avançada, o que necessariamente não é verdadeiro. Apesar disso, trata-se de estudo mais rápido e simples de ser realizado que o estudo de coorte, que seria o ideal. A amostra de conveniência pode não ser representativa da populaçãoalvo, principalmente quando se trabalha com amostras pequenas. No presente estudo, a amostra final representou $76,0 \%$ da população-alvo, o que reduz o viés potencial da amostra de conveniência.

Em relação ao instrumento em si, questionários autoaplicados tendem a superestimar os escores, uma vez que podem ser influenciados pela desejabilidade social no momento de seu preenchimento. Além disso, a EEJ, apesar de ser um dos instrumentos mais utilizados para avaliação de empatia, é genérica, não sendo específica para o atendimento pediátrico, que possui peculiaridades em relação às outras especialidades. $\mathrm{O}$ fato de que os valores encontrados nesse estudo foram muito semelhantes àqueles que utilizaram a EEJ no atendimento em geral e de que parte dos estudantes estavam cursando outra disciplina no momento da aplicação dos questionários, podem indicar que foi medida a empatia em relação ao atendimento em geral e não ao atendimento pediátrico. Contudo, a revisão da literatura não identificou nenhum instrumento autoaplicado que avalie a atitude empática do médico ou estudante de Medicina em relação ao paciente pediátrico. Existe um instrumento para avaliação da empatia médica pelo paciente, a escala Consultation and Relational Empathy (CARE), que possui uma versão para pacientes adultos e uma para pediatria ${ }^{24,25}$. Ela avalia a capacidade do médico em compreender a situação do paciente, as circunstâncias familiares, as perspectivas, os sentimentos e a comunicação com os familiares. A escala CARE pediátrica, que ainda não foi adaptada para o Brasil, possui três versões:

- $5 \mathrm{Q}$ - para crianças de 7 a 11 anos;

- $10 \mathrm{Q}$ - para indivíduos com idade entre 12 e 16 anos; e
- $\quad$ 10Q PAIS - para pais de crianças com 0 a 6 anos ou para crianças incapazes de preencher.

Apesar de suas limitações, até onde se sabe, esse é o primeiro estudo que procurou avaliar a empatia de estudantes de Medicina em relação a pacientes pediátricos no Brasil, o que lhe confere caráter inédito. Além disso, apresenta bom número de participantes, teve pequeno percentual de perdas e as associações observadas apresentam significância estatística e são plausíveis com os achados na literatura.

A empatia em pediatria é tema de grande relevância para a educação e a prática profissional e precisa ser mais estudada. Novos projetos nessa linha poderiam focar a adaptação transcultural e a validação da escala CARE pediátrica para o português e o desenvolvimento de questionário autoaplicado que avalie a atitude empática de médicos e estudantes de Medicina, utilizando essa escala como parâmetro de controle ou comparação externa.

\section{CONCLUSÕES}

Os níveis de empatia (escore global e diferentes domínios) observados neste estudo foram elevados, com exceção do domínio "capacidade de se colocar no lugar do outro". Não se observou relação entre escore global de empatia e seus diferentes domínios e o ano do curso médico.

\section{REFERÊNCIAS}

1. Luecke Jr PE. The history of pediatrics at Baylor University Medical Center. Proc (Bayl Univ Med Cent). 2004;17:56-60.

2. Gusson ACT, Lopes JC. Pediatria no século 21: uma especialidade em perigo. Rev. Paul. Pediatr. 2010;28:115-20.

3. Levetown M. Communicating with children and families: from everyday interactions to skill in conveying distressing information. Pediatrics. 2008;121:e1441-60.

4. Armelin CB, Wallau RA, Sarti CA, Pereira SR. A comunicação entre os profissionais de pediatria e a criança hospitalizada. Rev. bras. crescimento desenvolv. hum. 2005;15:45-54.

5. Goold SD, Lipkin Jr M. The doctor-patient relationship: challenges, opportunities, and strategies. J. gen. intern. med. 1999;14 Suppl 1:S26-33.

6. Honavar SG. Patient-physician relationship - Communication is the key. Indian j. ophthalmol. 2018;66:1527-8.

7. Davis MH. Measuring individual difference in emphaty: Evidence for a multidimensional approach. J Person Soc Phychol. 1983;44:113-26.

8. Hojat M, Mangione S, Nasca TJ, Rattner S, Erdmann JB, Gonnella JS, et al. An empirical study of decline in empathy in medical school. Med. educ. 2004;38:934-41.

9. Costa FD, Azevedo RCS. Empatia, relação médico-paciente e formação em medicina: um olhar qualitativo. Rev. bras. educ. med. 2010;34:261-9.

10. Aguiar P, Salgueira A, Frada T, Costa M. Empatia médica: tradução, validação e aplicação de um instrumento de medição. Ata do $X$ Congresso Internacional Galego-Português de Psicopedagogia. Braga: Universidade do Minho; 2009.

11. Hojat M, DeSantis J, Gonnella JS. Patient perceptions of clinician's empathy: measurement and psychometrics. J Patient Exp. 2017;4:78-83.

12. Hojat M, DeSantis J, Shannon SC, Mortensen LH, Speicher MR, Bragan L, et al. The Jefferson Scale of Empathy: a nationwide study of measurement properties, underlying components, latent variable

REVISTA BRASILEIRA DE EDUCAÇÃO MÉDICA

$744(3): \mathrm{e} 089 ; 2020$ 
structure, and national norms in medical students. Adv Health Sci Educ Theory Pract. 2018;23:899-920.

13. Hojat M, Vergare MJ, Maxwell K, Brainard G, Herrine SK, Isenberg GA, et al. The devil is in the third year: a longitudinal study of erosion of empathy in medical school. Acad. med. 2009;84:1182-91.

14. Nascimento HCF, Ferreira Júnior WA, Silva AMTC, Carvalho IGM, Bastos GCFC, Almeida RJ. Análise dos níveis de empatia de estudantes de Medicina. Rev. bras. educ. med. 2018;42:150-8.

15. Gabarra LM, Crepaldi MA. A comunicação médico-paciente pediátrico-família na perspectiva da criança. Psicol. argum. 2011;29:209-18

16. Neumann M, Edelhauser F, Tauschel D, Fischer MR, Wirtz M, Woopen C, et al. Empathy decline and its reasons: a systematic review of studies with medical students and residents. Acad. med. 2011;86:996-1009.

17. Moreto G, Blasco PG. A erosão da empatia nos estudantes de medicina: um desafio educacional. Rev Bras Med. 2012;69:7-12.

18. Filho ED, Dias RB, Toledo Júnior A. Ações para a retomada do ensino da humanização nas escolas de medicina. Rev. bras. educ. med. 2018;42:14-28.

19. Paro HB, Daud-Gallotti RM, Tiberio IC, Pinto RM, Martins MA. Brazilian version of the Jefferson Scale of Empathy: psychometric properties and factor analysis. BMC med. educ. 2012;12:73.

20. Kataoka HU, Koide N, Ochi K, Hojat M, Gonnella JS. Measurement of empathy among Japanese medical students: psychometrics and score differences by gender and level of medical education. Acad. med. 2009;84:1192-7.

21. Magalhães E, Salgueira AP, Costa P, Costa MJ. Empathy in senior year and first year medical students: a cross-sectional study. BMC med. educ. 2011;11:52.
22. Hojat M, Gonnella JS, Nasca TJ, Mangione S, Vergare M, Magee M. Physician empathy: definition, components, measurement, and relationship to gender and specialty. Am. j. psychiatr. 2002;159:1563-9.

23. Esquerda $M$, Yugueroa $O$, Viñasa J, Pifarré J. La empatía médica, ¿nace o se hace? Evolución de la empatía en estudiantes de medicina. Aten. prim. 2016;48:8-14.

24. Arigliani M, Castriotta L, Pusiol A, Titolo A, Petoello E, Peressut AB, et al. Measuring empathy in pediatrics: validation of the Visual CARE measure. BMC pediatr. 2018;18:57.

25. Place MA, Murphy J, Duncan EA, Reid JM, Mercer SW. A preliminary evaluation of the Visual CARE Measure for use by Allied Health Professionals with children and their parents. J. child health care. 2016;20:55-67.

\section{CONTRIBUIÇÃO DOS AUTORES}

Naiara Silva Cosmo de Araújo e Antonio Toledo Júnior participaram da elaboração do estudo, da interpretação dos dados, da elaboração do artigo, da revisão do artigo e da aprovação da versão final. Naiara Silva Cosmo de Araújo realizou a coleta de dados, e Antonio Toledo Júnior realizou a análise estatística.

\section{CONFLITO DE INTERESSES}

Os autores declaram não haver conflito de interesses.

\section{ENDEREÇO PARA CORRESPONDÊNCIA}

Antonio Toledo Júnior. Rua Califórnia, 570/501, Sion, Belo Horizonte, MG, Brasil. CEP: 30315-500.

E-mail: antonio.toledo@unifenas.br 